\title{
Genetic and Pharmacological Inhibition of Rheb1-mTORC1 Signaling Exerts Cardioprotection against Adverse Cardiac Remodeling in Mice
}

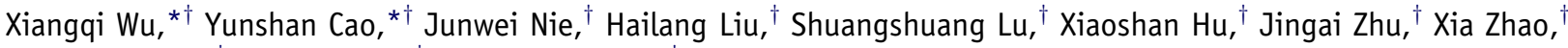 \\ Jiandong Chen, ${ }^{\ddagger}$ Xiaohu Chen, ${ }^{\ddagger}$ Zhongzhou Yang, ${ }^{\dagger}$ and Xinli $\mathrm{Li}^{*}$
}

\begin{abstract}
From the Department of Cardiology,* the First Affiliated Hospital of Nanjing Medical University, Nanjing; the Ministry of Education Key Laboratory of Model Animal for Disease Study, ${ }^{\dagger}$ Model Animal Research Center, Nanjing University, Nanjing; and the Jiangsu Provincial Traditional Chinese Medicine Hospital Affiliated to Nanjing University of Traditional Chinese Medicine, ${ }^{\ddagger}$ Nanjing, China
\end{abstract}

\author{
Accepted for publication \\ February 7, 2013 \\ Address correspondence to \\ Zhongzhou Yang, Ph.D., Ministry \\ of Education Key Laboratory of \\ Model Animal for Disease Study, \\ Model Animal Research Center, \\ Nanjing University, 12 Xuefu Rd. \\ Pukou, Nanjing 210061, China; or \\ Xinli Li, M.D., Department of \\ Cardiology, the First Affiliated \\ Hospital of Nanjing Medical \\ University, 300 Guangzhou Rd, \\ Nanjing 210029, China. E-mail: \\ zhongzhouyang@nju.edu.cn or \\ xinli3267@yeah.net.
}

\begin{abstract}
A previous study indicated that Rheb1 is required for mammalian target of TOR complex 1 (mTORC1) signaling in the brain. However, the function of Rheb1 in the heart is still elusive. In the present study, we deleted Rheb1 specifically in cardiomyocytes and found that reduced Rheb1 levels conferred cardioprotection against pathologic remodeling in myocardial infarction (MI) and pressure overload (transverse aortic constriction) mouse models. Cardiomyocyte apoptosis was reduced and mTORC1 activity was suppressed in cardiomyocyte Rheb1-deletion mice, suggesting that Rheb1 regulates mTORC1 activation in myocardium. Furthermore, we demonstrated that astragaloside IV (As-IV) could inhibit mTORC1, and As-IV treatment displayed similar protection against MI and transverse aortic constriction as Rheb1 genetic inhibition. This study indicates that Rheb1 is essential for mTORC1 activation in cardiomyocytes and suggests that targeting Rheb1-mTORC1 signaling, such as by As-IV treatment, may be an effective therapeutic method for treating patients with adverse cardiac remodeling after MI and hypertrophy. (Am J Pathol 2013, 182: 2005-2014; http://dx.doi.org/10.1016/ j.ajpath.2013.02.012)
\end{abstract}

Congestive heart failure (CHF) is a leading cause of morbidity and mortality worldwide. CHF is commonly caused by pathologic cardiac remodeling after a wide spectrum of cardiovascular diseases, such as hypertension and myocardial infarction (MI). ${ }^{1-3}$ Pathologic cardiac remodeling refers to changes in geometry, mass, volume, and function of the heart after injury to the ventricles. Major hallmarks of this remodeling include enlarged cardiomyocyte size, the buildup of extracellular matrix, and increased cardiomyocyte apoptosis. ${ }^{4}$ Although myocardial remodeling is compensatory initially, it can become maladaptive and eventually lead to $\mathrm{CHF}$ if left untreated. Therefore, the relief of adverse myocardial remodeling is a critical therapeutic strategy to treat $\mathrm{CHF}$. Despite the widespread use of $\beta$-blockers and inhibitors of the renin-angiotensin-aldosterone system clinically in the past few decades, the incidence of $\mathrm{CHF}$ still remains high, highlighting the need for additional understanding of remodeling events.
In recent years, protein synthesis involved in the remodeling process has been given remarkable attention. One important molecule related to protein synthesis is the mechanistic target of rapamycin (TOR) complex 1 or mammalian target of TOR complex 1 (mTORC1) that is involved in cell growth, proliferation, and differentiation. mTORC1 activity is strongly stimulated by upstream Rheb, whereas tuberous sclerosis complex $1 / 2$ negatively regulates mTORC 1 by inhibition of Rheb activity.

There are two Rheb isoforms, termed Rheb1 and Rheb2, in mice. ${ }^{6}$ Deletion of Rhebl in mice results in embryonic

\footnotetext{
Supported in part by National Key Basic Research Program of China grants 2011CB943904 and 2012CB966602; National Natural Science Foundation of China grants 31071282, 91019002, 31130037, 81170201, and 81273943 (Z.Y., X.L., and X.C.); and the Open Research Fund of State Key Laboratory of Bioelectronics, Southeast University.
} 


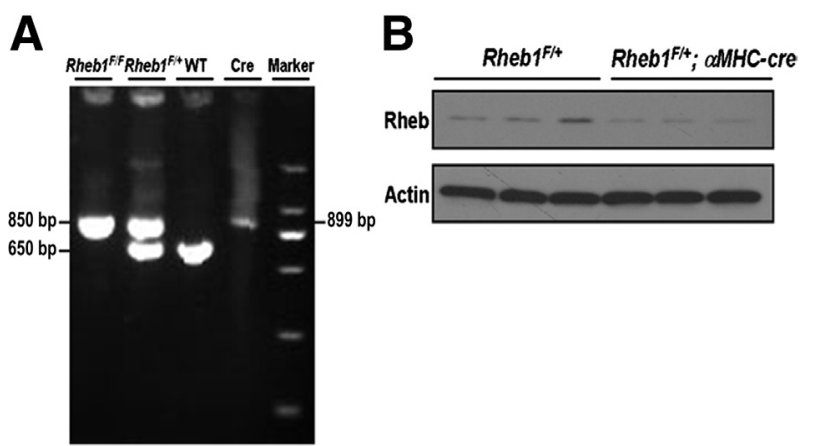

Figure 1 Generation of cardiomyocyte-specific Rheb1-deletion mice. A: Genotyping of mice by PCR. B: Western blot analysis of Rheb in the heart. WT, wild type.

lethality between embryonic day (E) 10.5 and E11.5, whereas loss of Rheb2 in mice does not affect embryonic development, and mice could survive to adulthood without apparent defects. ${ }^{6}$ In addition, Rheb 1 has been shown to be essential for mTORC1 signaling. ${ }^{6}$
To understand the function of Rheb in the heart, we deleted Rhebl in cardiomyocytes through Cre-mediated excision in mice. Half loss of Rheb1 conferred cardiac protection against pathologic heart remodeling resulting from $\mathrm{MI}$ and pressure overload. Cardiac mTORC1 signaling was disrupted in Rhebldeficient mice. These results demonstrated that Rheb mediates mTORC1 signaling in the heart and that Rheb-mTORC1 signaling is involved in pathologic heart remodeling. Meanwhile, this study suggested that inhibition of Rheb-mTORC1 signaling might be an effective strategy for treating pathologic heart remodeling.

Rapamycin is a potent mTOR inhibitor. ${ }^{7}$ However, recent studies have revealed the adverse effects of rapamycin, such as body weight loss, increased risks of infection and cancer, and diabetes-like symptoms. ${ }^{7-11}$ Therefore, it is clinically significant to screen a new drug targeting mTORC1 signaling with mild adverse effects. In the present study, we found that astragaloside IV (As-IV), extracted from Chinese medicine Astragalus membranaceus (Fisch) Bge, could inhibit mTORC1 activity in different cell lines, such as

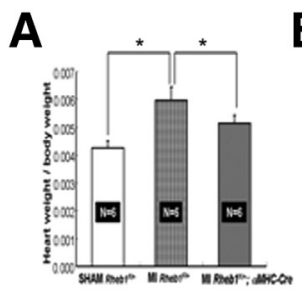

$\mathbf{F}$

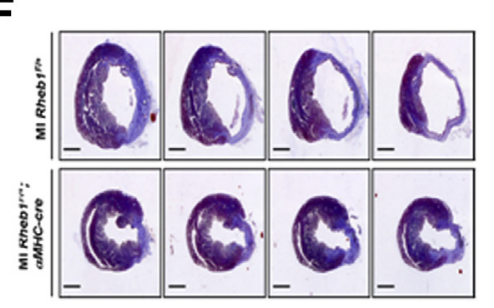

$\mathbf{J}$
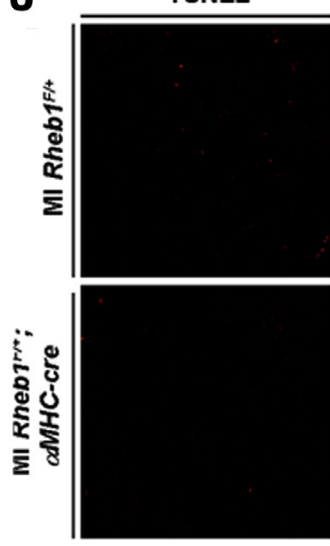

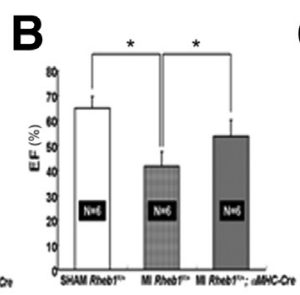

G

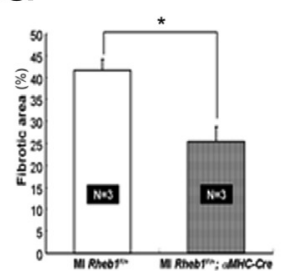

To-Pro-3
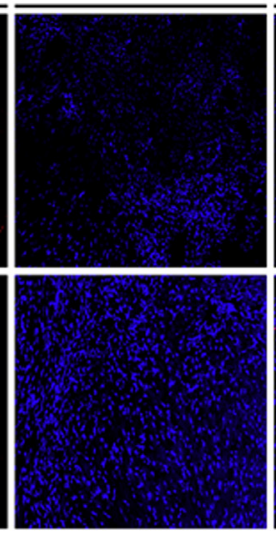

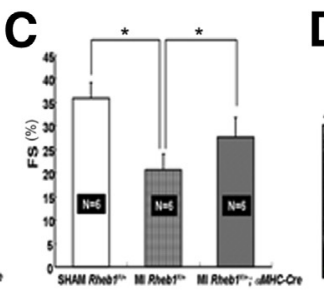

H

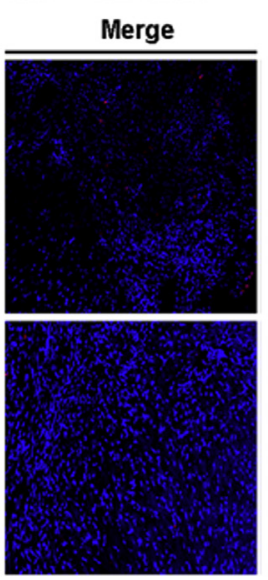

D
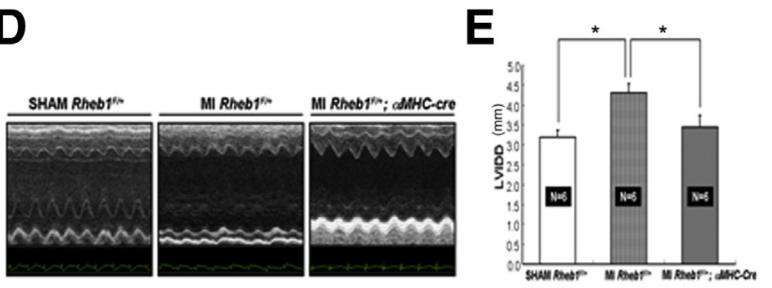

I

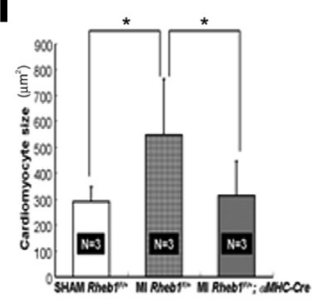

K

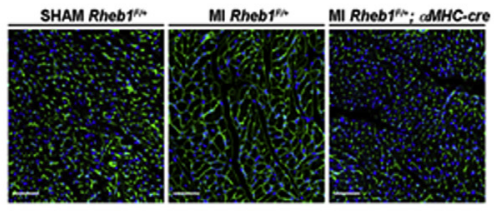

$\mathbf{L}$
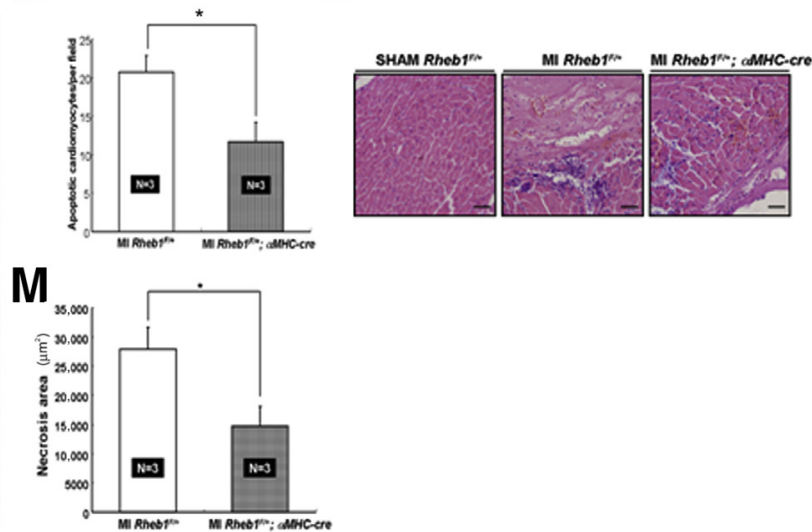

Figure 2 Loss of Rheb1 ameliorates cardiac dysfunction and aberrant heart remodeling after MI. A: Ratio of heart weight to body weight. B-E: Echocardiography measurement. F: Masson's staining was used to display fibrotic areas (in blue). G: Quantitation of fibrotic area ( $n=3$ ). H: Wheat germ agglutinin staining was used to display the structure and size of cardiomyocytes. Sections were from papillary muscle. I: Quantitation of cross areas of cardiomyocytes $(n=3)$. J: TUNEL assay. Apoptotic cells are in red. Sections were taken from the border zone of the infarct area. K: Quantitation of apoptotic cells $(n=3)$. L: H\&E staining showed necrosis and inflammation in the border zone of the infarct area 3 days after MI surgery. M: Quantitation of necrosis area $(n=3)$. EF, ejection fraction; FS, fractional shortening; LVIDD, left ventricular internal diastolic diameter; SHAM, mice after sham operation. Data are given as means \pm SEM. ${ }^{*} P<0.01$. Scale bars: $50 \mu \mathrm{mol} / \mathrm{L}(\mathbf{H}) ; 30 \mu \mathrm{mol} / \mathrm{L}(\mathbf{L})$. 
A

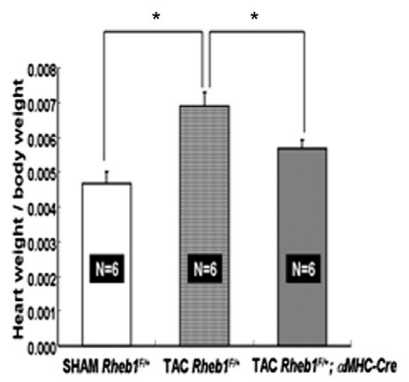

D D
B

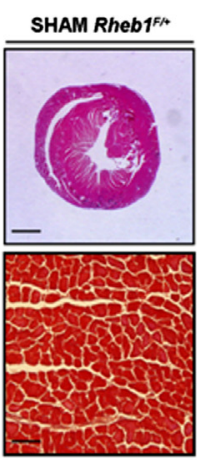

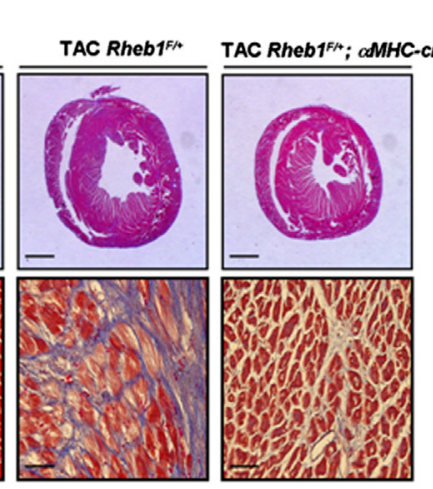
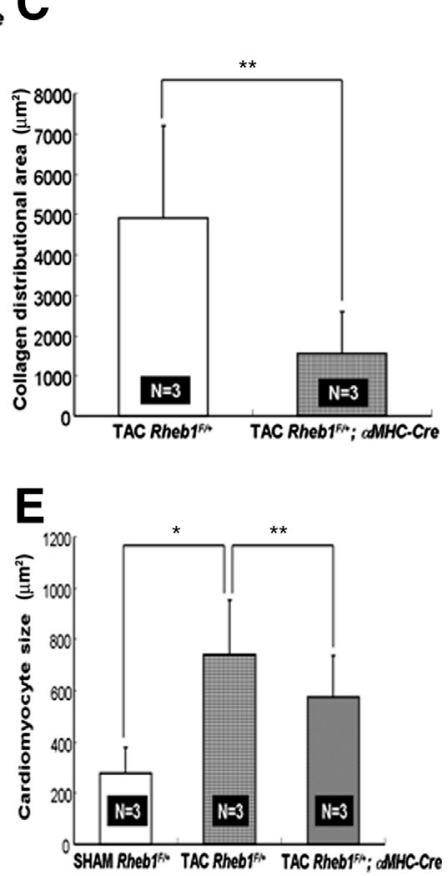

Figure 3 Deletion of Rheb1 represses cardiac hypertrophy induced by pressure overload. A: Ratio of heart weight to body weight. B: H\&E staining was used to show the cross section of heart size, and Masson's staining was used to display fibrotic areas (in blue). C: Quantitation of collagen distribution ( $n=3$ ). D: Wheat germ agglutinin staining of structure and size of cardiomyocytes. Sections were from papillary muscle. E: Quantitation of cross areas of cardiomyocytes ( $n=3)$. SHAM, mice after sham operation. Data are given as means \pm SEM. ${ }^{*} P<0.01,{ }^{*} P<<0.05$. Scale bars: $1 \mathrm{mmol} / \mathrm{L}$ (B, upper panels); $40 \mu \mathrm{m}$ (B, lower panels); $50 \mu \mathrm{m}($ (D).

HeLa and HEK293. Moreover, As-IV was shown to effectively alleviate pathologic heart remodeling and to reduce mTORC1 signaling in mouse models.

\section{Materials and Methods}

Mice

Mice with a C57BL/6 genetic background were housed in groups with 12-hour dark/light cycles and with free access to food in accordance with the regulations on mouse welfare and ethics of Nanjing University (Nanjing, China). All the procedures were conducted with relevant authority approval. Ras homolog enriched in brain (Rheb1)-floxed mice were as described previously and were maintained on a C57BL/6 genetic background. ${ }^{6}$ To delete Rhebl in cardiomyocytes, Rhebl-floxed mice were crossed with $\alpha$-myosin heavy chain $(\alpha M H C)-C r e$ mice, and the progenies were genotyped by PCR. DNA primers for Rhebl genotyping were as follows: $5^{\prime}$-GCCCAGAACATCTGTTCCAT- $3^{\prime}$ and 5'-GGTACCCACAACCTGACACC- $3^{\prime}$ to amplify wild-type and $5^{\prime}$ floxed alleles, respectively. PCR products for wild-type and floxed alleles are 650 and $850 \mathrm{bp}$, respectively (Figure 1A).

\section{Cell Culture and As-IV Treatment}

As-IV (98\% purity) was purchased from Nanjing Zelang Medical Technology Co. Ltd. (Nanjing, China).

$293 \mathrm{~T}$ cells and $\mathrm{HeLa}$ cells were grown at $37^{\circ} \mathrm{C}$ in Dulbecco's modified Eagle's medium supplemented with $10 \%$ fetal calf serum. These cells were treated with As-IV $(1 \mu \mathrm{mol} / \mathrm{L})$ or dimethyl sulfoxide and were collected at 36 and 48 hours for Western blot analysis. In parallel, 100 $\mathrm{nmol} / \mathrm{L}$ rapamycin was applied to these cells for 1 hour for mTOR inhibition.

Ventricular myocytes were prepared from mice (2 to 3 days after birth) with a C57BL/6 genetic background and cultured on Permanox chamber slides (Thermo Scientific Nunc, Waltham, MA) coated with $1 \%$ gelatin and $0.0015 \%$ laminin solution, as previously described. ${ }^{12}$ Afterward, these cells were treated with dimethyl sulfoxide, isoproterenol (ISO) $(10 \mu \mathrm{mol} / \mathrm{L})+$ dimethyl sulfoxide, or ISO $(10 \mu \mathrm{mol} / \mathrm{L})+\mathrm{As}-\mathrm{IV}(1 \mu \mathrm{mol} / \mathrm{L})$ for 2 days. After treatment, these cells were collected for immunofluorescence staining and Western blot analysis.

\section{Mouse Models of MI and TAC and Administration of As-IV}

MI and transverse aortic constriction (TAC) were generated following a method reported previously in mice. ${ }^{13,14}$ Briefly, male mice aged 8 to 12 weeks weighing 25 to $30 \mathrm{~g}$ were anesthetized i.p. with 0.4 to $0.75 \mathrm{mg} / \mathrm{g}$ of body weight tribromoethanol [Avertin (2,2,2-tribromoethanol; Sigma-Aldrich, St. Louis, MO)]. An endotracheal tube was introduced into the trachea, and a volume-cycled rodent respirator (model 683; Harvard Apparatus, Holliston, MA) provided positive pressure ventilation at 2 to $3 \mathrm{~mL}$ per cycle with a respiratory rate of 120 cycles per minute. For MI surgery, the thoracic cavity was opened and ligation of the left anterior descending coronary artery was performed 


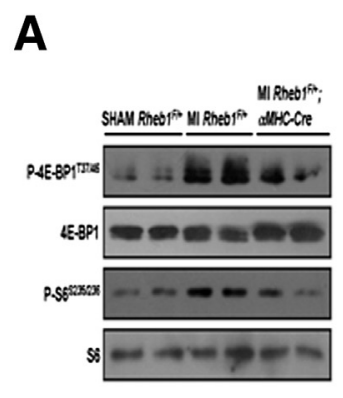

E

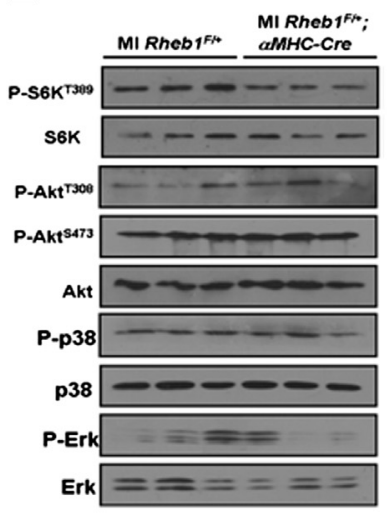

B

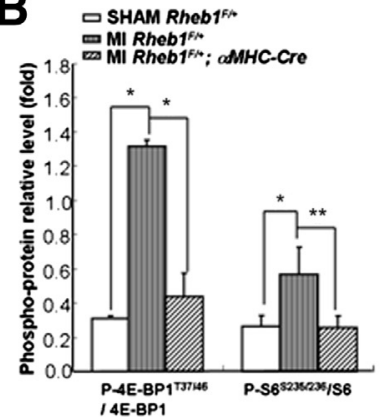

F

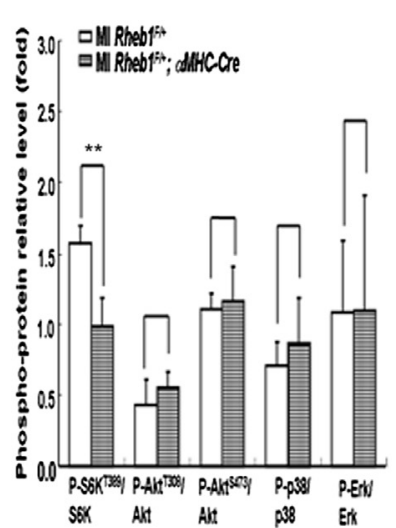

C

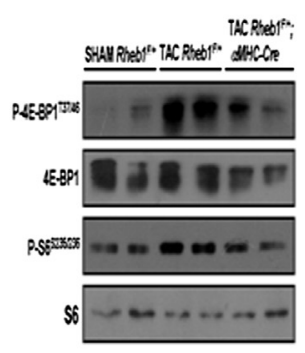

G

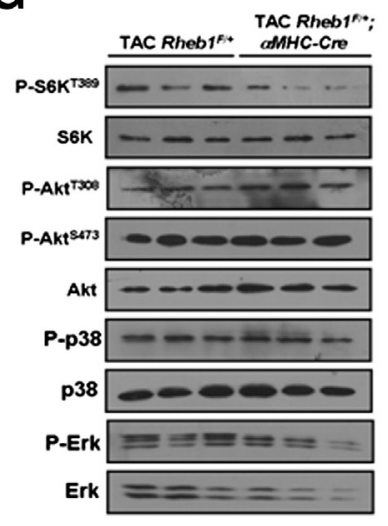

D

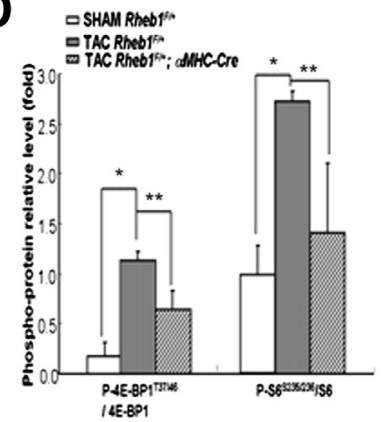

H

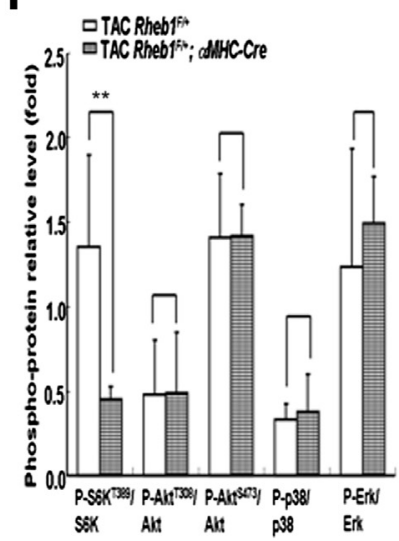

Figure 4 Cardiac ablation of Rheb1 impairs mTORC1 signaling. A: Heart tissues were dissected from a remote area for Western blot analysis of S6 and 4E-BP1 phosphorylation (P) in mice with MI. B: Quantitative study $(n=3)$. C: Western blot analysis of S6 and 4E-BP1 phosphorylation in heart tissues of mice after TAC. D: Quantitative study $(n=3)$. E: Western blot analysis of S6K, Akt, p38, and ERK phosphorylation in heart tissues (remote area) of MI mice. F: Quantitative study $(n=3)$. G: Western blot analysis of S6K, Akt, p38, and ERK phosphorylation in heart tissues of TAC mice. H: Quantitative study ( $n=3)$. SHAM, mice after sham operation. Data are given as means \pm SEM. ${ }^{*} P<0.01,{ }^{*} * P<0.05$.

using a 7-0 silk suture 3 to $4 \mathrm{~mm}$ from the tip of the left auricle. The chest was closed using a continuous 6-0 prolene suture, followed by a 4-0 polyester suture to close the skin. TAC surgery was performed by tying a 7-0 polypropylene suture around the transverse aorta against a 27 -gauge needle to yield a narrowing $0.4 \mathrm{~mm}$ in diameter.

As-IV was administrated to mice i.p. at a dose of $2 \mathrm{mg} / \mathrm{kg}$ of body weight for 21 days after MI and TAC surgery.

\section{Echocardiography}

Echocardiography was performed using a Vevo 770 UBM system (VisualSonics Inc., Toronto, ON, Canada) that possesses a single-element mechanical transducer with a center frequency of $30 \mathrm{MHz}$ and a frame rate of $30 \mathrm{~Hz}^{7}$ The spatial resolution of B-mode imaging was approximately 115 $\mu \mathrm{m}$ (lateral) by approximately $55 \mu \mathrm{m}$ (axial). Mice were anesthetized with Avertin as described for MI surgery. The body temperature of mice was monitored using a rectal thermometer and was maintained between $36^{\circ} \mathrm{C}$ and $38^{\circ} \mathrm{C}$. The heart rate was maintained between 350 and 450 beats $/ \mathrm{min}$. After measurement, cardiac output values, such as ejection fraction, fractional shortening, and left ventricular internal diastolic diameter, were calculated according to the guidelines accompanying the Vevo 660 UBM system. ${ }^{7}$

\section{Western Blot Analysis}

Heart tissues were collected from the MI remote area in the left ventricle. Tissue lysates were prepared in lysis buffer $(20 \mathrm{mmol} / \mathrm{L}$ Tris, $150 \mathrm{mmol} / \mathrm{L} \mathrm{NaCl}, 10 \%$ glycerol, $20 \mathrm{mmol} / \mathrm{L}$ glycerophosphate, $1 \% \mathrm{NP} 40,5 \mathrm{mmol} / \mathrm{L}$ EDTA, $0.5 \mathrm{mmol} / \mathrm{L} \mathrm{EGTA}, 1 \mathrm{mmol} / \mathrm{L} \mathrm{Na}_{3} \mathrm{VO}_{4}, 0.5 \mathrm{mmol} / \mathrm{L}$ phenylmethylsulfonyl fluoride, $1 \mathrm{mmol} / \mathrm{L}$ benzamidine, $1 \mathrm{mmol} / \mathrm{L}$ dithiothreitol, $50 \mathrm{mmol} / \mathrm{L} \mathrm{NaF}$, and $4 \mu \mathrm{mol} / \mathrm{L}$ leupeptin, $\mathrm{pH}$ 8.0). Samples were resolved by $10 \%$ SDS-PAGE and were transferred to polyvinylidene difluoride membranes (Millipore, Billerica, MA). Membranes were blocked with Trisbuffered saline and Tween $20(50 \mathrm{mmol} / \mathrm{L}$ Tris, 150 $\mathrm{mmol} / \mathrm{L} \mathrm{NaCl}$, and $0.5 \mathrm{mmol} / \mathrm{L}$ Tween $20, \mathrm{pH} 7.5$ ) and then were incubated with primary antibodies overnight. The following antibodies were purchased from Cell Signaling Technology Inc. (Danvers, MA): Rheb (\#4935), S6 (\#2317), phosphor-S6 (S235/236) (\#2211), phosphor-S6 (S240/244) (\#2215), 4E-BP1 (\#9452), phosphor-4E-BP1 (T37/46) (\#9459), phosphor-4E-BP1(S65) (\#9451), p70S6K (\#9202), phospho-p70S6K (Thr389) (\#9206), total Akt (\#9272), phospho-Akt (Thr308) (\#9275), phospho-Akt (Ser473) (\#9271), extracellular signal-regulated kinase (ERK; \#9102), phospho-ERK (Tyr202/Tyr204) (\#9101), p38 (\#9212), and phospho-p38 (Thr180/Tyr182) (\#9211). Pan- 
A
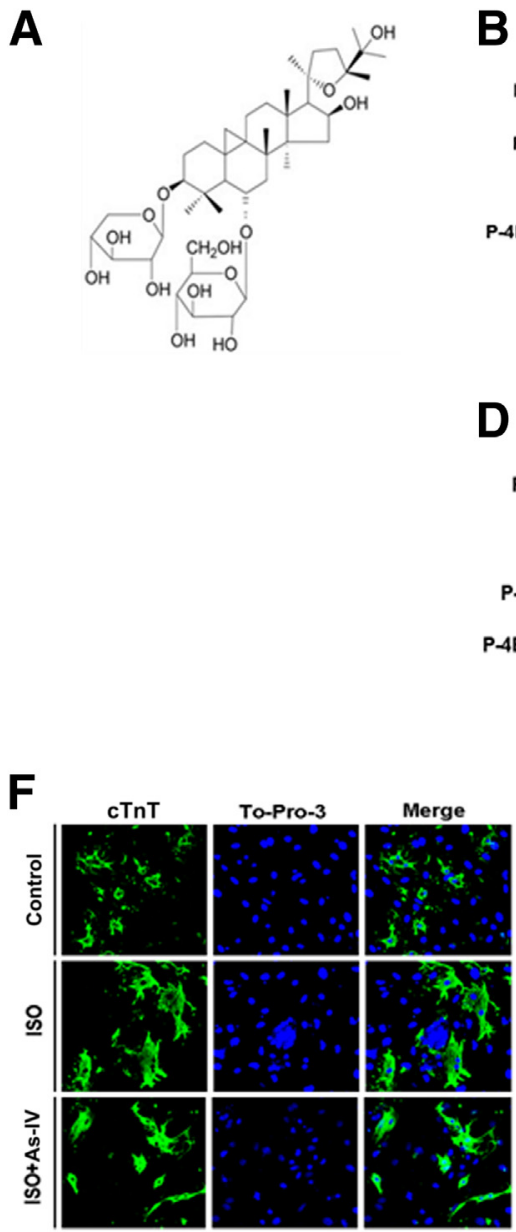

B

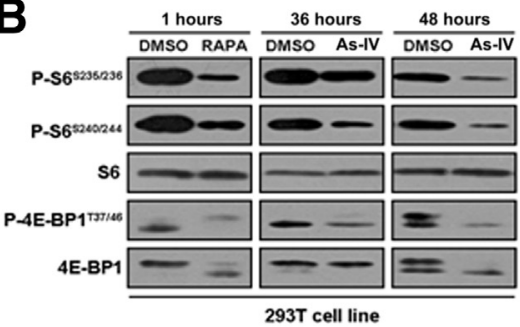

D

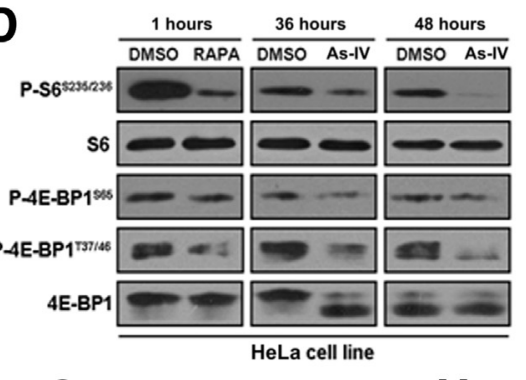

G
C

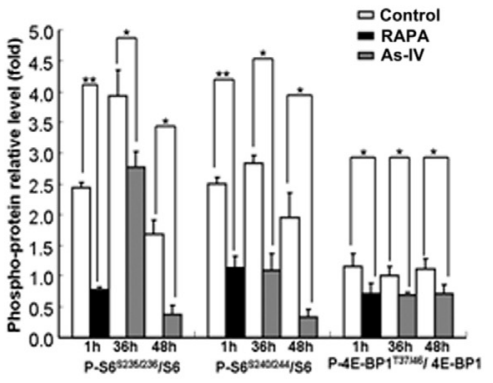

E

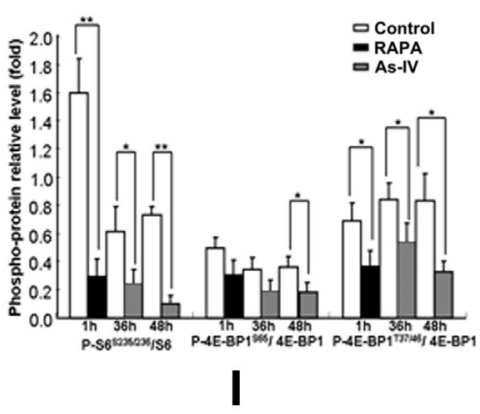

Figure 5 Identification of As-IV as a natural inhibitor of mTORC1 signaling. A: The structure of As-IV. B: 293T cells were incubated with rapamycin (RAPA) or As-IV and were collected for Western blot analysis. C: Quantitative study $(n=3)$. D: HeLa cells incubated with RAPA or As-IV were collected for Western blot analysis. E: Quantitative study $(n=3)$. F: Cardiac troponin T (cTnT) staining (green) of cardiomyocytes. G: Quantitative study $(n=3)$. H: Cardiomyocytes from young mice 2 to 3 days after birth incubated with As-IV and ISO were collected for Western blot analysis. I: Quantitative study $(n=3)$. Data are given as means \pm SEM. ${ }^{*} P<0.05,{ }^{* *} P<0.01$.

actin antibody (\#MS-1295-P0) and horseradish peroxidaselinked secondary antibodies (\#31460 and \#31430) were purchased from Thermo Scientific (Rockford, IL).

\section{Histologic Analysis and Immunofluorescence Staining}

H\&E and Masson's staining and immunofluorescence were performed as described previously. ${ }^{7,15,16}$ Briefly, heart samples were first washed with cold PBS and then were fixed in $4 \%$ paraformaldehyde at $4{ }^{\circ} \mathrm{C}$. The samples were processed successively by i) a 30-minute wash in PBS at $4{ }^{\circ} \mathrm{C}$; ii) 15 -minute incubations in $30 \%, 50 \%, 75 \%$, and $85 \%$ ethanol and then two 10-minute incubations in 95\% and $100 \%$ ethanol at room temperature; iii) three 10-minute incubations in xylene at room temperature; iv) a 20-minute incubation in paraffin/xylene (dilution $1: 1$ ) at $65^{\circ} \mathrm{C}$; and v) three 30-minute incubations in fresh paraffin at $65^{\circ} \mathrm{C}$. The processed samples were then embedded in paraffin and sectioned ( $6 \mu \mathrm{m}$ thick), and the sections were stained. Immunofluorescence staining was performed using anticardiac troponin $\mathrm{T}$ antibody and anti-wheat germ agglutinin antibody at $4{ }^{\circ} \mathrm{C}$ overnight. Fluorescence microscopy images were obtained using a research fluorescence microscope (Olympus America Inc., Center Valley, PA) equipped with a digital camera. Images were collected and recorded using Photoshop version 5.0 (Adobe Systems Inc., San Jose, CA) on an IBM R52 computer (IBM, Armonk, NY).

\section{TUNEL Assay}

TUNEL assay was performed as previously reported. ${ }^{7,15}$ Briefly, the sections were treated with $20 \mu \mathrm{g} / \mathrm{mL}$ of proteinase $\mathrm{K}$ and were incubated with terminal deoxynucleotidyl transferase and biotinylated deoxyuridine triphosphate.

\section{Statistical Analysis}

Data are presented as means \pm SEM values. For comparisons between two groups, statistical significance was determined using the unpaired two-tailed Student's $t$-test. A $P<0.05$ was considered statistically significant, and a $P<0.01$ was considered statistically very significant. 


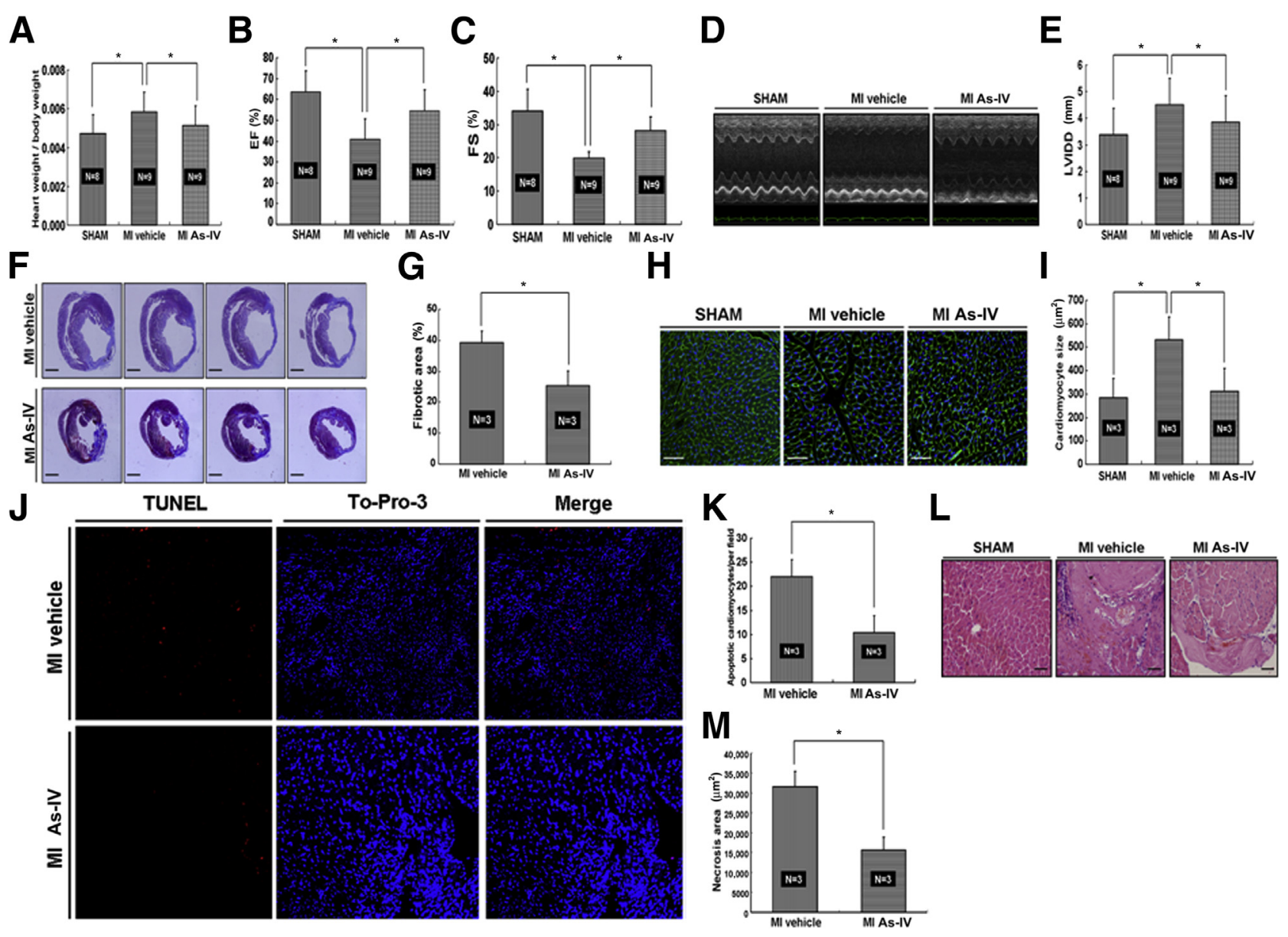

Figure 6 As-IV improves cardiac function and protected against MI. A: Ratio of heart weight to body weight. B-E: Echocardiography measurement. F: Masson's staining was used to display fibrotic areas (in blue). G: Quantitative study $(n=3)$. H: Wheat germ agglutinin staining was used to display the structure and size of cardiomyocytes. Sections were from papillary muscle. I: Quantitation of cross areas of cardiomyocytes $(n=3)$. J: TUNEL assay. Apoptotic cells are shown in red. Sections were taken from the border zone of the infarct area. K: Quantitative study $(n=3)$. L: H\&E staining was used to show necrosis and inflammation in the border zone of the infarct area 3 days after MI surgery. M: Quantitation of the necrosis area $(n=3)$. EF, ejection fraction; FS, fractional shortening; LVIDD, left ventricular internal diastolic diameter; SHAM, mice after sham operation. Data are given as means \pm SEM. ${ }^{\star} P<0.01$. Scale bars: $1 \mu \mathrm{m}(\mathbf{F}) ; 50 \mu \mathrm{m}(\mathbf{H}) ; 30 \mu \mathrm{m}(\mathrm{L})$.

\section{Results}

Generation of Cardiomyocyte-Specific Rheb1-Deletion Mice

Rhebl-floxed mice were as previously reported and were maintained in a C57/B6 genetic background. ${ }^{6}$ To delete $R h e b 1$ specifically in cardiomyocytes, female Rhebl-floxed mice $\left(R h e b 1^{F / F}\right)$ were crossed with male $\alpha M H C$-Cre mice to obtain Rhebl ${ }^{F /+}$; $\alpha M H C$-Cre mice. Mice were genotyped by PCR (Figure 1A), and loss of Rheb1 in heart tissue was confirmed by Western blot analysis. The results revealed that Rheb1 protein levels were significantly reduced in Rheb1-deletion mice compared with controls (Figure 1B).

\section{Loss of Rheb1 Ameliorates Cardiac Dysfunction and Aberrant Heart Remodeling after MI}

Disruption of both alleles of Rhebl in cardiomyocytes $\left(R h e b l^{F / F} ; \alpha M H C-C r e\right)$ resulted in mouse mortality at a young age (data not shown). However, half deletion of Rhebl in cardiomyocytes $\left(\right.$ Rhebl $1^{F /+} ; \alpha M H C$-Cre $)$ had little effect on heart function and mouse survival. To investigate the role of Rheb1 in cardiac protection, we first performed MI surgery in control and Rhebl-deletion mice to induce myocardial damage. Three weeks after MI, the heart weight to body weight ratio was increased significantly in control mice (Figure 2A). However, loss of Rheb1 profoundly reduced the heart weight to body weight ratio (Figure 2A). Echocardiography measurement indicated that heart systolic function was markedly impaired in control MI mice and well preserved in Rheb1-deletion mice (Figure 2, B and C). In addition, loss of Rhebl greatly reduced the left ventricular internal diastolic diameter compared with control MI mice (Figure 2, D and E). Histologic analysis displayed substantially reduced fibrosis area, cardiomyocyte size, and cell apoptosis in Rhebl-deletion mice compared with control MI mice (Figure 2, F-K). Finally, we investigated necrosis 3 days after MI and found that necrosis and inflammation in the border area was reduced in Rhebl- 

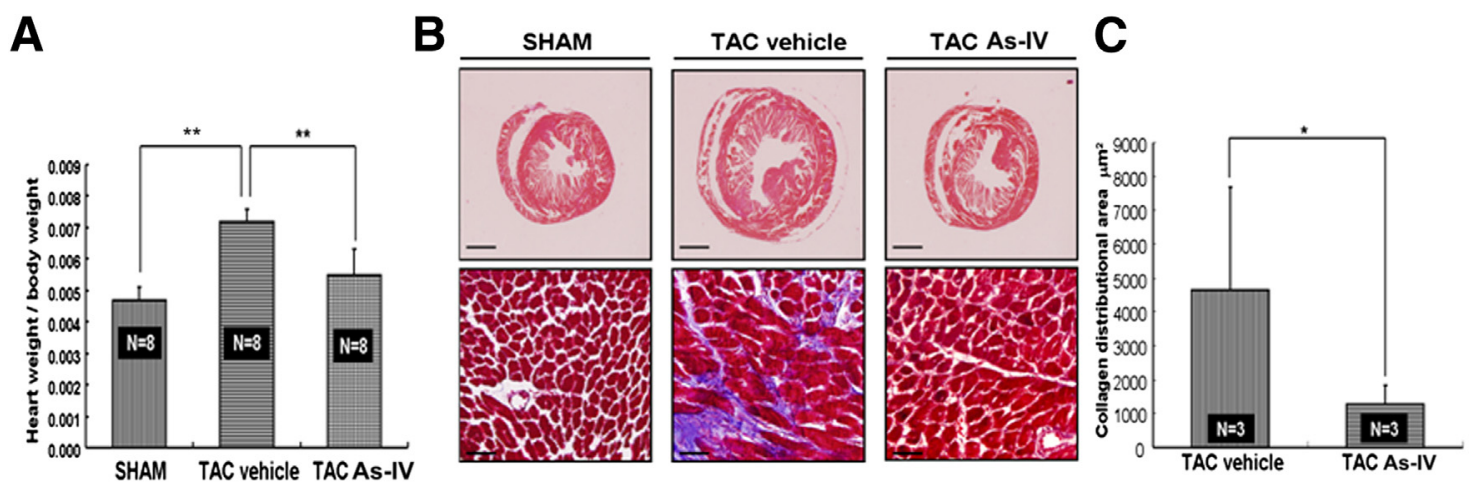

D
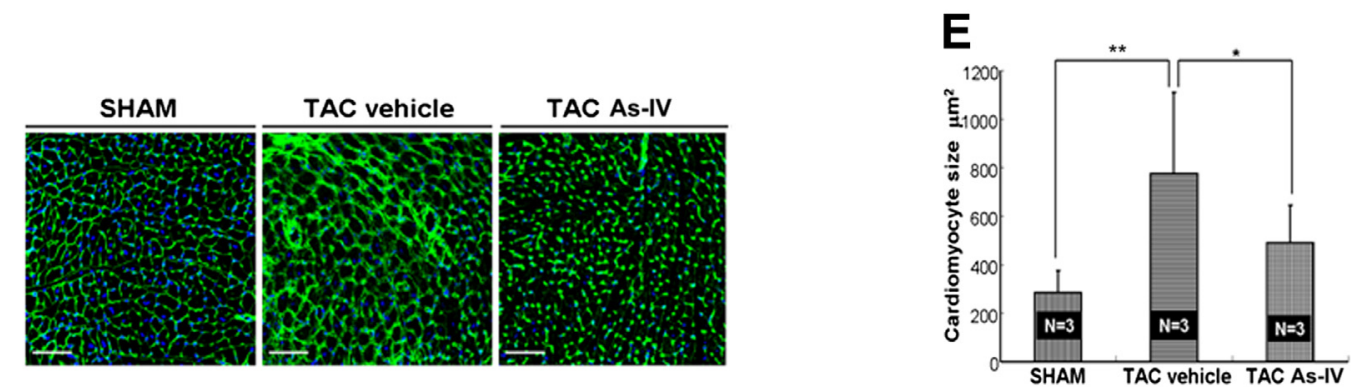

Figure 7 As-IV suppresses cardiac remodeling induced by TAC. A: Ratio of heart weight to body weight. B: H\&E staining was used to show the cross section of heart size, and Masson's staining was used to display fibrotic area (in blue). C: Quantitative study $(n=3)$. D: Wheat germ agglutinin staining was used to display the structure and size of cardiomyocytes. Sections were from papillary muscle. E: Quantitation of cross areas of cardiomyocytes ( $n=3$ ). SHAM, mice after sham operation. Data are given as means \pm SEM. ${ }^{*} P<0.05,{ }^{*} P<<0.01$. Scale bars: $1 \mathrm{mmol} / \mathrm{L}$ (B, upper panels); $40 \mu \mathrm{m}$ (B, lower panels); $50 \mu \mathrm{m}$ (D).

deletion mice compared with control MI mice (Figure 2, $\mathrm{L}$ and $\mathrm{M})$.

Taken together, these results indicated that genetic ablation of Rheb1 dramatically suppressed pathologic heart remodeling and improved heart function after MI. Thus, Rheb1 functions to protect against myocardial damage in the heart.

\section{Deletion of Rheb1 Represses Cardiac Hypertrophy Induced by Pressure Overload}

Next, we investigated the function of Rheb1 in heart hypertrophy. Control and Rhebl-deletion mice were challenged with TAC to induce heart hypertrophy. Three weeks after TAC surgery, cardiac hypertrophy was apparent in control mice but was significantly repressed in Rhebl-deletion mice (Figure 3, A and B). Masson's staining revealed extensive cardiac fibrosis in control mice, whereas it was hard to detect cardiac fibrosis in Rheb1-deletion mice (Figure 3, B and C). In control mice, TAC substantially increased cardiomyocyte size revealed by immunofluorescence staining, but this effect was less severe in Rheb1-deletion mice (Figure 3, D and E).

Collectively, these results indicated that Rheb1 was involved in pathologic remodeling and hypertrophic cardiomyopathy.

\section{Cardiac Ablation of Rheb1 Impairs mTORC1 Signaling}

We examined mTORC1 signaling in the heart tissue after MI and TAC surgery. Phosphorylation levels of 4E-BP1 and
S6, indicators of mTORC1 signaling, were profoundly enhanced in control mice after MI and TAC (Figure 4, A-D). In contrast, deletion of Rhebl markedly reduced the phosphorylation levels of 4E-BP1 and S6, indicating impaired mTORC1 signaling (Figure 4, A-D). Furthermore, we examined the signaling pathways of S6K, Akt, ERK, and $\mathrm{p} 38$. The results showed that phospho-S6K levels were significantly reduced in Rheb-deletion mice compared with control MI and TAC mice, whereas phosphorylation levels of Akt, ERK, and p38 were not changed (Figure 4, E-H).

\section{Identification of As-IV as a Natural Inhibitor of mTORC1 Signaling}

Rapamycin and its derivatives have been extensively studied, and they are well accepted as potent mTOR inhibitors. ${ }^{7}$ Nonetheless, recent studies indicated that these chemicals might cause severe adverse effects, such as glucose metabolic disorder, gastrointestinal inflammation, and immunosuppression. ${ }^{7-11}$ Therefore, it is necessary to search for better natural products to substitute for rapamycin and its derivatives for clinical application. We tested As-IV, a monomeric compound isolated from Chinese herbs that have been demonstrated to be effective in treating human cardiovascular diseases, and found that it could inhibit mTORC1 signaling; the structure of As-IV is shown in Figure 5A. Treatment of HEK293T and HeLa cells with As-IV significantly reduced 4E-BP1 and S6 phosphorylation (Figure 5, B-E). ISO treatment was shown to induce cardiomyocyte hypertrophy. ${ }^{17,18}$ We isolated cardiomyocytes 

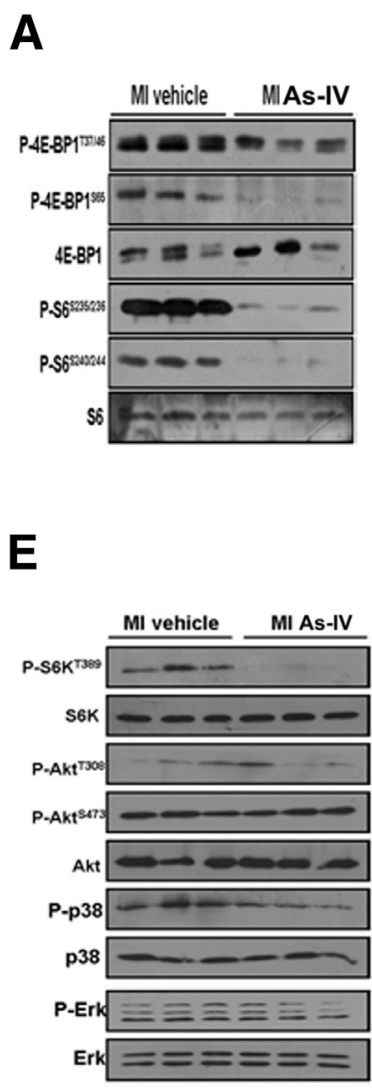

B

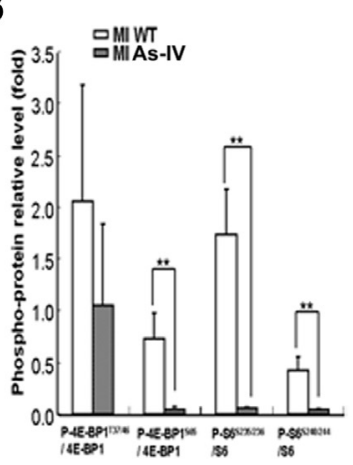

$\mathbf{F}$

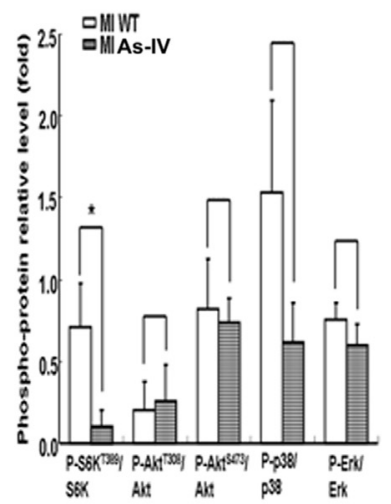

C

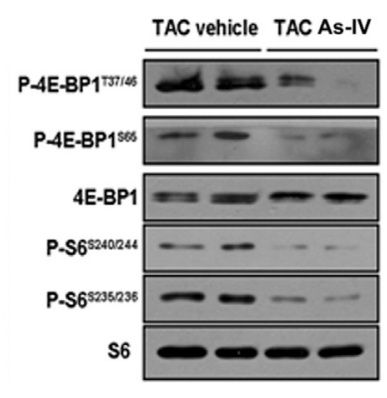

G

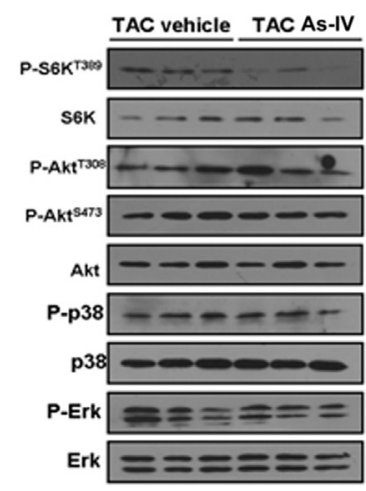

D

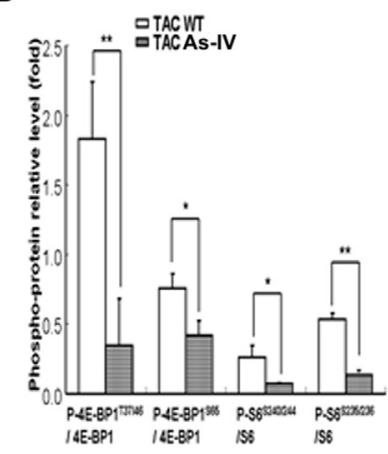

H

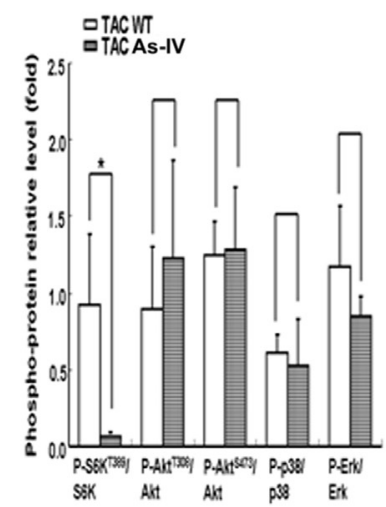

Figure 8 As-IV inhibits mTORC1 signaling in the heart. A: Heart tissues were dissected from a remote area for Western blot analysis of S6 and $4 \mathrm{E}-\mathrm{BP} 1$ phosphorylation (P) in mice with MI. B: Quantitative study $(n=3)$. C: Western blot analysis of S6 and 4E-BP1 phosphorylation in heart tissues of mice after TAC. D: Quantitative study $(n=3)$. E: Western blot analysis of S6K, Akt, p38, and ERK phosphorylation in heart tissues (remote area) of MI mice. F: Quantitative study $(n=3)$. G: Western blot analysis of S6K, Akt, p38, and ERK phosphorylation in heart tissues of TAC mice. H: Quantitative study ( $n=3)$. WT, wild type. Data are given as means \pm SEM. ${ }^{*} P<0.05,{ }^{*} P<0.01$.

from young mice 2 to 3 days after birth and cultured these cells. ISO treatment of these cells for 2 days caused hypertrophy (Figure 5, F and G). Co-treatment with ISO and As-IV significantly reduced cardiomyocyte hypertrophy (Figure 5, F and G). Western blot analysis showed that ISO treatment activated mTORC1 signaling, which was blocked by As-IV (Figure 5, $\mathrm{H}$ and $\mathrm{I})$.

\section{As-IV Improves Cardiac Function and Protects against MI}

Next, we tested the effects of As-IV in the MI mouse model. We first investigated whether As-IV had toxic effects in mice. Mice were treated with As-IV for 6 weeks. Afterward, body weight and heart function were measured, and the results indicated that As-IV did not impair heart function and reduce body weight (Supplemental Figure S1). After MI surgery, mice were administered As-IV for 21 days; the heart weight to body weight ratio was significantly reduced in As-IV-treated mice compared with vehicle-treated mice (Figure 6A). As-IV treatment also dramatically improved heart function as indicated by ejection fraction percentage and fractional shortening percentage (Figure 6, B and C). Meanwhile, heart dilatation was greatly suppressed after
As-IV administration (Figure 6, D and E). In addition, fibrotic area, cardiomyocyte hypertrophy, and cell apoptosis were markedly reduced in As-IV-treated mice compared with vehicle-treated mice (Figure 6, F-K). We also investigated necrosis 3 days after MI and found that necrosis and inflammation in the border area was reduced in As-IVtreated mice compared with control MI mice (Figure 6, L and $\mathrm{M}$ ). These results demonstrated that As-IV effectively improved heart function and protected against adverse cardiac remodeling after MI.

\section{As-IV Suppresses Cardiac Remodeling Induced by TAC}

We further investigated whether As-IV could inhibit cardiac remodeling induced by TAC. Mice underwent TAC surgery and were subsequently administered As-IV for 21 days. Figure 7A shows that the heart weight to body weight ratio was smaller in As-IV-treated mice than in vehicle-treated mice. Histologic analysis revealed less hypertrophic myocardium and reduced cardiomyocyte size in As-IV-treated mice compared with controls (Figure 7, B-E). Taken together, these results indicated that As-IV effectively suppressed cardiac remodeling induced by TAC. 
As-IV Inhibits mTORC1 Signaling in the Heart

Heart tissues were collected from As-IV- and vehicletreated mice 21 days after MI and TAC for Western blot analysis. mTORC1 signaling was analyzed, and the results showed that As-IV treatment substantially blocked mTORC1 signaling, as indicated by reduced 4E-BP1 and S6 phosphorylation levels (Figure 8, A-D). Furthermore, we examined the signaling pathways of S6K, Akt, ERK, and p38. The results showed that phospho-S6K levels were significantly reduced in As-IV-treated mice compared with control MI and TAC mice, whereas phosphorylation levels of Akt, ERK, and p38 were not changed (Figure 8, E-H).

\section{Discussion}

In the present study, we provided the first genetic evidence that ablation of Rhebl improved heart function and suppressed pathologic heart remodeling after MI and TAC. mTORC1 signaling activation was repressed by loss of Rhebl in the heart after MI and TAC, suggesting that Rhebmediated mTORC1 signaling is involved in aberrant heart remodeling. Subsequently, we reported the identification of As-IV, a natural product, as a potent inhibitor against mTORC1 signaling and that As-IV rendered a beneficial effect on cardiac protection against MI and pressure overload. This study not only elucidated the function of Rheb1 in cardiac mTORC1 signaling and remodeling but also provided supporting evidence for the clinical applications of As-IV to treat cardiomyopathy.

Rapamycin and its derivatives are potent inhibitors against mTOR signaling. However, severe adverse effects of rapamycin and its derivatives limit their clinical application and prompt the search for better substitutes. ${ }^{7-11}$ Astragalus membranaceus Bge, a Chinese herb, has been widely prescribed to improve heart function. ${ }^{19-21}$ However, the underlying mechanisms in treating cardiovascular disease are elusive. In this study, we identified As-IV, a monomeric compound isolated from Astragalus membranaceus $\mathrm{Bge}$, as a novel inhibitor against mTORC1 signaling. The results of this study suggest that Astragalus membranaceus Bge executes cardiac protection, in part through inhibition of mTORC1 signaling.

Previously, it was reported that $S 6 k 1 / S 6 k 2$ DKO mice showed no heart phenotype. ${ }^{22}$ However, overexpression of $S 6 k$ in cardiomyocytes substantially increased heart size, leading to hypertrophy in mice. Thus, it seems that loss of function of S6K has little effect on heart morphology but that gain of function does. Recently, Condorelli and colleagues $^{23}$ deleted $m$ Tor in cardiomyocytes and found dilated cardiomyopathy. This study demonstrated that loss of mTor impaired heart morphology and function. They also found that the protein levels of 4EBP1 were markedly elevated, suggesting that 4EBP1 is the major downstream target of mTOR in regulating heart morphology and function. However, in another study by Shen et al, ${ }^{24}$ it was found that mTOR kinase-dead transgenic mice did not manifest morphologic changes. In mTOR kinase-dead transgenic mice, the protein levels of 4EBP1 were not significantly changed, and this may be the reason why these mice did not show the heart morphologic phenotype as mTor-deletion mice displayed. Furthermore, we found that half loss of Rhebl $\left(\right.$ Rhebl $\left.^{+/-}\right)$had little effect on Akt S473 phosphor-

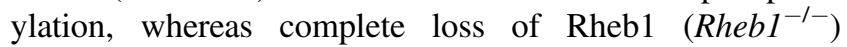
significantly enhanced the phosphorylation levels of Akt S473, probably through an mTORC1-Akt-negative feedback mechanism. ${ }^{7}$

In summary, we demonstrated that activation of mTORC1 is mediated by Rheb in the heart, which is involved in cardiac remodeling. Genetic and pharmacologic inhibition of Rheb-mTORC1 renders beneficial effects against cardiomyopathy caused by $\mathrm{MI}$ and pressure overload. As-IV, a natural product, can inhibit mTORC1 signaling and may be developed as a potential drug for treating pathologic heart remodeling and other diseases related to mTORC1 hyperactivation.

\section{Acknowledgment}

We are grateful to Dr. Bo Xiao (Sichuan University of China) for providing the Rhebl-floxed mice.

\section{Supplemental Data}

Supplemental material for this article can be found at http://dx.doi.org/10.1016/j.ajpath.2013.02.012.

\section{References}

1. Cheng S, Vasan RS: Advances in the epidemiology of heart failure and left ventricular remodeling. Circulation 2011, 124:e516-e519

2. Deedwania PC: The key to unraveling the mystery of mortality in heart failure: an integrated approach. Circulation 2003, 107:1719-1721

3. Velagaleti RS, Pencina MJ, Murabito JM, Wang TJ, Parikh NI, D'Agostino RB, Levy D, Kannel WB, Vasan RS: Long-term trends in the incidence of heart failure after myocardial infarction. Circulation 2008, 118:2057-2062

4. Kehat I, Molkentin JD: Molecular pathways underlying cardiac remodeling during pathophysiological stimulation. Circulation 2010, 122:2727-2735

5. Laplante M, Sabatini DM: mTOR signaling in growth control and disease. Cell 2012, 149:274-293

6. Zou J, Zhou L, Du XX, Ji Y, Xu J, Tian J, Jiang W, Zou Y, Yu S, Gan L, Luo M, Yang Q, Cui Y, Yang W, Xia X, Chen M, Zhao X, Shen Y, Chen PY, Worley PF, Xiao B: Rheb1 is required for mTORC1 and myelination in postnatal brain development. Dev Cell 2011, 20:97-108

7. Di R, Wu X, Chang Z, Zhao X, Feng Q, Lu S, Luan Q, Hemmings BA, Li X, Yang Z: S6K inhibition renders cardiac protection against myocardial infarction through PDK1 phosphorylation of Akt. Biochem J 2012, 441:199-207

8. Chhajed PN, Dickenmann M, Bubendorf L, Mayr M, Steiger J, Tamm M: Patterns of pulmonary complications associated with sirolimus. Respiration 2006, 73:367-374 
9. Weischer M, Rocken M, Berneburg M: Calcineurin inhibitors and rapamycin: cancer protection or promotion? Exp Dermatol 2007, 16: 385-393

10. Tataranni T, Biondi G, Cariello M, Mangino M, Colucci G, Rutigliano M, Ditonno P, Schena FP, Gesualdo L, Grandaliano G: Rapamycin-induced hypophosphatemia and insulin resistance are associated with mTORC2 activation and Klotho expression. Am J Transplant 2011, 11:1656-1664

11. Alfonso F, Moreno R, Vergas J: Fatal infection after rapamycin eluting coronary stent implantation. Heart 2005, 91:e51

12. Kapiloff MS, Schillace RV, Westphal AM, Scott JD: mAKAP: an A-kinase anchoring protein targeted to the nuclear membrane of differentiated myocytes. J Cell Sci 1999, 112(pt 16):2725-2736

13. Ahn D, Cheng L, Moon C, Spurgeon H, Lakatta EG, Talan MI: Induction of myocardial infarcts of a predictable size and location by branch pattern probability-assisted coronary ligation in C57BL/6 mice. Am J Physiol Heart Circ Physiol 2004, 286:H1201-H1207

14. Patten RD, Pourati I, Aronovitz MJ, Alsheikh-Ali A, Eder S, Force T, Mendelsohn ME, Karas RH: 17 Beta-estradiol differentially affects left ventricular and cardiomyocyte hypertrophy following myocardial infarction and pressure overload. J Card Fail 2008, 14: 245-253

15. Yang ZZ, Tschopp O, Di-Poi N, Bruder E, Baudry A, Dümmler B, Wahli W, Hemmings BA: Dosage-dependent effects of Akt1/protein kinase Balpha (PKBalpha) and Akt3/PKBgamma on thymus, skin, and cardiovascular and nervous system development in mice. Mol Cell Biol 2005, 25:10407-10418

16. Yang ZZ, Tschopp O, Hemmings-Mieszczak M, Feng J, Brodbeck D, Perentes E, Hemmings BA: Protein kinase B alpha/Akt1 regulates placental development and fetal growth. J Biol Chem 2003, 278: 32124-32131
17. Pesce L, Comellas A, Sznajder JI: Beta-adrenergic agonists regulate Na-K-ATPase via p70S6k. Am J Physiol Lung Cell Mol Physiol 2003, 285:L802-L807

18. Simm A, Schluter K, Diez C, Piper HM, Hoppe J: Activation of p70(S6) kinase by beta-adrenoceptor agonists on adult cardiomyocytes. J Mol Cell Cardiol 1998, 30:2059-2067

19. Liu ZL, Liu ZJ, Liu JP, Yang M, Kwong J: Herbal medicines for viral myocarditis. Cochrane Database Syst Rev 2010, CD003711

20. Zhang N, Wang XH, Mao SL, Zhao F: Astragaloside IV improves metabolic syndrome and endothelium dysfunction in fructose-fed rats. Molecules 2011, 16:3896-3907

21. Lv L, Wu SY, Wang GF, Zhang JJ, Pang JX, Liu ZQ, Xu W, Wu SG, Rao JJ: Effect of astragaloside IV on hepatic glucose-regulating enzymes in diabetic mice induced by a high-fat diet and streptozotocin. Phytother Res 2010, 24:219-224

22. McMullen JR, Shioi T, Zhang L, Tarnavski O, Sherwood MC, Dorfman AL, Longnus S, Pende M, Martin KA, Blenis J, Thomas G, Izumo S: Deletion of ribosomal S6 kinases does not attenuate pathological, physiological, or insulin-like growth factor 1 receptorphosphoinositide 3-kinase-induced cardiac hypertrophy. Mol Cell Biol 2004, 24:6231-6240

23. Zhang D, Contu R, Latronico MV, Zhang J, Rizzi R, Catalucci D, Miyamoto S, Huang K, Ceci M, Gu Y, Dalton ND, Peterson KL, Guan KL, Brown JH, Chen J, Sonenberg N, Condorelli G: MTORC1 regulates cardiac function and myocyte survival through 4E-BP1 inhibition in mice. J Clin Invest 2010, 120:2805-2816

24. Shen WH, Chen Z, Shi S, Chen H, Zhu W, Penner A, Bu G, Li W, Boyle DW, Rubart M, Field LJ, Abraham R, Liechty EA, Shou W: Cardiac restricted overexpression of kinase-dead mammalian target of rapamycin (mTOR) mutant impairs the mTOR-mediated signaling and cardiac function. J Biol Chem 2008, 283:13842-13849 